\title{
Una experiencia efímera: Enrique de Benito y el Instituto de Estudios Penales de la Universidad de Valencia (1928-1930)
}

\author{
An Ephemeral Experience: Enrique de Benito \\ and the Institute of Criminal Studies \\ at the University of Valencia (1928-1930)
}

\author{
María Pilar Hernando Serra* \\ Universitat de València \\ ORCID ID: 0000-0001-9382-6394
}

$\begin{array}{ll}\text { Recibido: } & 15 / 07 / 2020 \\ \text { Aceptado: } & 24 / 09 / 2020\end{array}$

DOI: https://doi.org/10.20318/cian.2020.5795

Resumen: El auge de la Criminología en la segunda mitad del siglo XIX y en los primeros treinta años del XX dio lugar a una pequeña pero importante escuela de criminólogos españoles. El éxito del correccionalismo, junto con la extensión de las teorías de la Escuela Positiva italiana, dio lugar a la creación del Laboratorio de Criminología en Madrid, y la Escuela de Criminología para funcionarios o enseñanzas complementarias al Derecho Penal. En Valencia, la creación del Instituto de Estudios Penales, impulsado por el profesor
Abstract: The rise of Criminology in the second half of the 19th century and in the first thirty years of the 20th century gave rise to a small but important school of Spanish criminologists. The success of correctionalism, together with the extension of the theories of the Italian Positive School, gave rise to experiences such as the Criminology Laboratory in Madrid, and the Criminology School for civil servants or complementary teachings to Criminal Law. In Valencia, the creation of the Institute of Criminal Studies, promoted by Professor En-

"pilar.hernando@uv.es 
Enrique De Benito fue una de esas experiencias. De corta trayectoria, el Instituto, con un plantel de profesores multidisciplinar y con un programa que abarcaba los conocimientos básicos de la Criminología, fue el antecedente del actual Instituto de Criminología de Valencia. Palabras clave: criminología, universidad, Valencia, correccionalismo, positivismo. rique De Benito, was one of those experiences. With a short history, the Institute, with a multidisciplinary staff of teachers and with a program that covered the basic knowledge of Criminology, was the antecedent of the current Institute of Criminology of Valencia.

Keywords: Criminology, University, Valencia, Correctionalism, Positivism.

En 1835, el coronel Manuel Montesinos y Molina asumía la dirección del presidio correccional de Valencia. Tan solo un año antes se había dictado la Ordenanza General de Presidios del Reino, ordenanza que Montesinos transformó en una práctica humanitaria sobre el penado, basada en la idea de la corrección. Muy bien podríamos decir que el sistema "progresivo" en la aplicación de la pena que él inició fue la primera manifestación práctica, el germen en España, de lo que luego sería la Ciencia de la Criminología. Cultivada por juristas, médicos, psicólogos o antropólogos, ninguno de ellos podrá, a partir de ese momento, dejar de hacer referencia en el desarrollo de la misma a la experiencia de la prisión de Valencia llevada a cabo por este singular militar ${ }^{1}$.

Casi un siglo después, la ciudad de Valencia volvería a estar unida a la Ciencia Criminológica. En 1909, en el marco de las actividades culturales y científicas que se habían organizado con ocasión de la Exposición Regional de Valencia, se celebró el primer Congreso Penitenciario Español. A esas alturas, la Criminología estaba ampliamente desarrollada no solo en Europa y América, sino también en España. La Escuela de Criminología de Madrid, creada en 1903, había supuesto la confirmación definitiva de un importante grupo, especialmente de juristas, pero no solo, dedicados al desarrollo y aplicación de la Criminología. También en la Universidad había hecho su aparición, en algunos planes de estudio -en doctorado especialmente-, y en actividades organizadas por algunos catedráticos, más a título personal que oficial. Sin embargo, en universidades como la valenciana la Criminología, por aquellas fechas, brillaba por su ausencia. No será hasta que arribe a nuestra universidad como profesor de Derecho Penal Enrique de Benito y de la Llave que la Criminología volverá a estar presente nuevamente en Valencia.

${ }^{1}$ La Revista de Estudios Penitenciarios dedicó un número monográfico en 1962 sobre la figura del coronel Montesinos. En dicho número se recogen, además de estudios diversos sobre su persona y "su sistema progresivo", sus propias reflexiones sobre su experiencia en la prisión de Valencia, así como el informe que dirigió al ministerio tras la aprobación del Código Penal de 1848 y los efectos que las penas tan largas recogidas en el Código podían producir en el sistema que él había practicado en Valencia, con tan buenos resultados. Revista de Estudios Penitenciarios, 159 (1962). 


\section{Algunos apuntes sobre la Criminología como enseñanza profesional}

Como sabemos, la moderada Ley de Instrucción Pública de 1857 abordó la ordenación de la enseñanza en su totalidad, después de unos cuantos años ya de andadura liberal -incluidas tres constituciones-. Además de regular las enseñanzas primaria y secundaria, se refería esta ley a tres tipos de enseñanza superior: la universitaria, la profesional y técnica, y otras calificadas genéricamente de superiores. Correspondían estas últimas a enseñanzas como la ingeniería de caminos, canales y puertos, ingenierías de minas, montes, agrónomos, industriales, notariado, diplomática y bellas artes; y clasificadas como enseñanzas profesionales y técnicas, las de comercio, veterinaria, náutica, maestros de obras, aparejadores, agrimensores y maestros de primera enseñanza. Sin entrar a valorar la bondad o no de la norma o su efectividad real, respecto a la enseñanza universitaria la ley de Moyano tuvo, en lo esencial, una larga vida. Y ello fue así fruto del especial interés que los gobiernos de uno y otro signo ponían en la universidad como institución formadora de los profesionales que, entre otras cosas, debían nutrir las instituciones fundamentales del todavía naciente estado contemporáneo ${ }^{2}$. Sin embargo, no merecieron idéntica atención las otras enseñanzas superiores. Aunque en algunos periodos se intentó impulsar su establecimiento -la formación de aquellas profesiones que se consideraban esenciales para el desarrollo del país-, lo bien cierto es que hasta el último tercio del siglo XIX solo fueron objeto de proyectos efímeros, sin dotación suficiente, ni económica ni de personal. Fue la realidad que se imponía la que obligó a crear centros de enseñanza no universitaria que tuvieran continuidad: escuelas de comercio, industriales, escuelas de agricultura, de administración, de bellas artes... Todas ellas necesarias para formar a los profesionales que necesitaban las urbes, cada vez más desarrolladas; los nuevos mercados, cada vez más amplios; o la Administración del Estado, cada vez más crecida.

Conforme crecía la Administración del Estado, la formación de sus funcionarios comenzaba a ser un problema que había que resolver. Es por ello por lo que, siguiendo el modelo francés, se constituyeron escuelas de Administración insertadas en las facultades de Derecho -incluso como una especialidad de la carrera-. Después, esta formación se integraría y se convertiría en una disciplina más de la carrera. Pues bien, en el estudio de la administración, uno de los ámbitos donde se insistió a finales de siglo sobre la adecua-

\footnotetext{
${ }^{2}$ Mariano Peset y José Luis Peset, La Universidad española (siglos XVIII y XIX): Despotismo ilustrado y revolución liberal, (Madrid: Taurus, 1974).
} 
da formación de los funcionarios fue en el sistema penitenciario. Fernando Cadalso y Concepción Arenal se convirtieron, con sus críticas y peticiones, en el azote de un sistema carcelario todavía anclado en el Antiguo Régimen y muy resistente a los cambios legislativos que se habían ido aplicando a lo largo del siglo. De esta manera, se hizo evidente la necesidad de una buena formación de aquellos funcionarios que debían ayudar al estado a cumplir una de sus funciones más delicadas: la función de punir.

A la par que se hacía evidente para algunos la necesidad de esta formación especial de los funcionarios, crecía en España el desarrollo de la Criminología como ciencia auxiliar del Derecho Penal desarrollada en relación al sistema penitenciario y al tratamiento del delincuente en el ámbito carcelario ${ }^{3}$. Adquiría, por lo tanto, un eminente carácter práctico. El éxito del correccionalismo de Röeder gracias, a su vez, al predominio de las teorías de Krause, favoreció la idea de la finalidad correctiva de la pena. Y esta solo se conseguiría con unos funcionarios con amplios conocimientos en psicología, sociología o antropología, capaces de entender al delincuente en toda su complejidad. La Criminología, pues, se entendía como una enseñanza profesional y práctica que ponía su atención y estaba dirigida a uno de los elementos humanos del sistema carcelario: los funcionarios de prisiones.

Pero muy pronto, el interés por la Criminología se insertó de lleno también en la Universidad. Especialmente los penalistas se interesaron por las corrientes criminológicas que venían de Europa. La confluencia del correccionalismo con las teorías y crítica a algunos aspectos de la Escuela Positiva italiana dieron lugar al éxito del correccionalismo tutelar o "Derecho Penal Protector", como lo llamaba Pedro García Dorado Montero ${ }^{4}$. Sus seguidores

${ }^{3}$ El devenir de la disciplina está unido en sus comienzos con el desarrollo en nuestro país de la legislación penitenciaria. Legislación que en resumidas cuentas tendría como objeto de atención tres elementos a lo largo de este periodo. En primer lugar, hubo un fuerte debate sobre el modelo carcelario, la estructura de las prisiones. En segundo lugar, o paralelamente a esta cuestión, el régimen disciplinario y su adscripción a la administración civil y su progresiva separación del ministerio de la Guerra. Por último, la formación especializada de los funcionarios de prisiones. Isabel Ramos Vázquez, "La Administración Civil Penitenciaria: militarismo y administrativismo en los orígenes del estado de Derecho", Anuario de Historia del Derecho Español, 82 (2012): 471-520; de la misma autora, La reforma penitenciaria en la historia contemporánea española, (Madrid: Dykinson, 2013).

${ }^{4}$ Sebastián Martín, "Penalística y penalistas españoles a la luz del principio de la legalidad (1874-1944)", Quaderni Fiorentini per la storia del pensiero giuridico moderno, 36 (2007): 503-609, 520-542. José María Hernández Díaz, "Nuevo derecho penal versus pedagogía correccional en Dorado Montero", en El largo camino hacia una educación inclusiva: la educación especial y social del siglo XIX a nuestros días: XV Coloquio de Historia de la Educación, 
lo plasmarían con la creación en 1899 del Laboratorio de Criminología en el seno de la Universidad de Madrid y un poco más tarde, del Seminario de Criminología ${ }^{5}$. Estos dos últimos proyectos, inspirados en el ideario institucionista, tuvieron como protagonistas a Francisco Giner de los Ríos, Luis Simarro, Constancio Bernaldo de Quirós y Rafael de Salillas 6 . Dos años más tarde, en 1901, se creaba la cátedra de doctorado de Antropología Criminal en la Universidad de Madrid. Esta cátedra fue ocupada en un primer momento por Félix Pío de Aramburu y Zuloaga, hasta ese momento catedrático de Derecho Penal en Oviedo. Más tarde la ocuparía Quintiliano Saldaña. Por supuesto, no solo cátedras o seminarios, sino un abundante número de revistas y otras publicaciones, que fueron el soporte tanto de estudios de carácter científico como de cuestiones relativas al sistema penitenciario, desde el punto de vista puramente jurídico o administrativo ${ }^{7}$. Todo este proceso confluyó en la creación de la Escuela de Criminología en $1903^{8}$. Esta se configuraba como una escuela especializada para la formación de los funcionarios de prisio-

Pamplona-Iruñea, 29, 30 de junio y 1 de julio de 2009, coords. María Reyes Berruezo Albéniz y Susana Conejero López, 2 vols., (Pamplona: Universidad Pública de Navarra, 2009), 125-132.

${ }^{5}$ El Laboratorio impartió dos cursos de 1899 a 1900, con profesores como el propio Giner, Bernaldo de Quirós, Cuello Calón, Simarro, Aguilaniedo o Salillas. Sobre el Laboratorio de Criminología y los profesores que en él impartieron, Alfonso Serrano Gómez, Historia de la Criminología en España, (Madrid: Dykinson, 2000), 449-460. También, Alfonso Serrano Maíllo, Un estudio sobre la formación de la Criminología española (1903-1978). Desarrollo, hostigamiento y dimensión simbólica, (Navarra: Aranzadi, 2018).

${ }^{6}$ Bernaldo de Quirós, además fue fundador y colaborador del Instituto de Reformas Sociales y subdirector de política agraria en el Ministerio de Salud y Previsión Social durante la República, entre otros servicios. Constancio Cassá Bernaldo de Quirós, Una pluma en el exilio. Los artículos publicados por Constancio Bernaldo de Quirós en República Dominicana, (Santo Domingo: Archivo General de la Nación, 2009). Yvan Lissorgues, "Pensar la psicología en español en la segunda mitad del siglo XIX. (Luis Simarro Lacabra, Urbano González Serrano, Leopoldo Alas, Clarín)”, en La filosofía y las lenguas de la península ibérica: Actas de las VIII y IX Jornadas Internacionales de Hispanismo filosófico. Barcelona, 2007, Santander, 2009, eds. José Luis Mora García, Ramón Emilio Mandado Gutiérrez, Gemma Gordo Piñar, Marta Nogueroles Jové, (Madrid: Fundación Ignacio Larramendi, 2010), 395-416. Por su parte, Rafael de Salillas fue director de la Prisión Celular de Madrid y el primer director de la Escuela de Criminología en Madrid. José Antón Oneca, "Don Rafael Salillas", Anuario de Derecho Penal y Ciencias Penales, 27, fasc. II (1974): 205-220.

${ }^{7}$ Revista de Prisiones (1894-1908); Revista Penitenciaria (1904-1908); Progreso Penitenciario. Revista de disciplina penal (1913-1927); La Policía Científica (1913-1914); Mundo Penal (1912-1917); etc. Jorge Alberto Núñez, Fernando Cadalso y la reforma penitenciaria, (Madrid: Universidad Carlos III de Madrid, 2014), 62.

${ }^{8}$ Siendo ministro de Gracia y Justicia, José Canalejas hizo la propuesta de una Escuela especial para funcionarios. Luis Gargallo Vaamonde, Desarrollo y destrucción del sistema liberal de prisiones en España. De la Restauración a la guerra civil, Tesis doctoral, (Universidad de Castilla-La Mancha, 2014), 114-115. 
nes, pero a la que se daba acceso también a estudiantes universitarios como ampliación y complemento de las enseñanzas de otras facultades y carreras que requirieran conocimientos criminológicos ${ }^{9}$. El objeto de atención ya no estaba solo en el delito, sino que pasaba a ponerse el acento en el delincuente; por otro lado, variaba el método de análisis donde ya no era suficiente el razonamiento jurídico, en favor de la experimentación científica y el método inductivo. Para la formación de los alumnos de la Escuela se articulaba un plan de estudios basado en asignaturas entonces consideradas complementarias a la Ciencia penal como la Antropología o la Sociología criminal, la Antropometría o la Psicología y la Estadística ${ }^{10}$. Su enseñanza debía tener un destacado carácter práctico, no solo teórico. En ella participaron, probablemente, lo más representativo de la naciente Criminología española: pedagogos como Manuel Bartolomé Cossío o Isidro Céspedes Mac-Crohon; psicólogos como Luis Simarro Lacabra o Manuel Hilario Ayuso; médicos como Federico Olóriz; antropólogos como Manuel Antón Ferrándiz o penalistas como Luis Jiménez de Asúa, Mariano Ruiz-Funes, Quintiliano Saldaña, Manuel López-Rey, Jaime Masaveu, José Guallart o Federico de Castejón Martínez de Arizala impartieron materias como Derecho Penal, Penología, Biopsicología criminal o Sociología Criminal y Estadística. La Escuela, como sabemos, fue suprimida por Miguel Primo de Rivera en 1926, para volver a recrearse en 1932 bajo el nombre de Instituto de Estudios Penales, siendo directora de prisiones Victoria Kent. En el bienio conservador volvió a denominarse Escuela de Criminología, restableciéndose como Instituto de Estudios Penales en 1936. En la zona republicana el Instituto siguió funcionando hasta $1938^{11}$.

Derecho Penal y Criminología en la Facultad de Derecho de Valencia: el profesor Enrique de Benito

La asignatura de Derecho Penal no era una materia con una gran tradición en los estudios de Derecho, como sí lo eran materias que entroncaban con las enseñanzas que ya se había impartido en las universidades medievales de Leyes y Cánones. Asignaturas como Derecho Civil, Derecho Romano, Derecho Canónico o incluso Economía Política, encontraban una tradición jurídica que

\footnotetext{
${ }^{9}$ Art. 2 del R. D. de 12 de marzo de 1903. Gaceta de Madrid, núm. 72, p. 1071.

${ }^{10}$ Rectificación realizada el 15 de marzo de 1903. Gaceta de Madrid, núm. 74, p. 1107.

${ }^{11}$ Se conserva el presupuesto de la escuela para el año 1939, fechado en enero, en Barcelona. Centro Documental de la Memoria Histórica, (en adelante, CDMH), P.S.- MADRID, caja 1.067, exp. 23.
} 
se alargaba hasta las épocas del Antiguo Régimen. Pero la enseñanza del Derecho Penal unida, como otras materias, a su codificación la convertía en una asignatura novedosa. Desde que comenzaron las primeras reformas de planes de estudio liberales la materia penal no había tenido individualidad propia. Había sido enseñada junto a otras materias como el derecho administrativo, el derecho civil o el derecho mercantil. Con la reforma de Gamazo en 1883 pasó a convertirse en la asignatura de Derecho Penal y Procedimiento criminal y un año después, con la reforma de Pidal Mon -apenas introducía cambios-, en Derecho Penal ${ }^{12}$. La reforma de Gamazo diseñó la carrera de Derecho con un curso preparatorio, donde se cursaban materias generales que se impartían en la facultad de Filosofía, y cuatro cursos de Derecho, cursándose en tercero la asignatura de Derecho Penal -o cuarto, si contamos el del preparatorio-.

Las reformas continuaron en los primeros años del siglo XX, dejando la carrera, estrictamente, en cinco años de Derecho -se extinguía para siempre el preparatorio-. Por una real orden de 31 de agosto de 1911 se decretaba que las asignaturas de Historia del Derecho y de Derecho Penal debían tener un carácter eminentemente práctico. La influencia de la Institución Libre de Enseñanza -después de más cuarenta años de existencia-, así como las nuevas corrientes pedagógicas que llegaban, sobre todo, de Francia y Alemania no pasaban desapercibidas, a pesar de la resistencia y fuerza que todavía ejercía la universidad "oficial" ${ }^{13}$. Lo cierto es que algunos catedráticos buscaban otros métodos más experimentales y enriquecedores para ejercer su docencia. Existía una tendencia favorable a la enseñanza práctica, también en el derecho, porque como se decía, "su misión es, en definitiva, preparar para la vida. ¿Cómo no han de serlo las enseñanzas profesionales como el derecho?" ${ }^{14}$. En otro orden de cosas, el R. D. de 21 de mayo de 1919 de autonomía universitaria preveía, por ejemplo, la posibilidad de que cada universidad creara en su seno centros, escuelas o colegios para el complemento de la enseñanza. Práctica y extensión de la enseñanza fueron, pues, los modelos que se repitieron en las reformas de aquellos años. Y esta tendencia fue en aumento y favoreció, además, la aparición de materias complementarias como las que se aprobaron el 7 de octubre de 1921 para la asignatura de Derecho

${ }^{12}$ Sobre la asignatura de Derecho penal en la facultad de Derecho de Valencia durante estos años, Yolanda Blasco Gil, La Facultad de Derecho de Valencia durante la Restauración (1875-1900), (Valencia: Universitat de València, 2000).

${ }^{13}$ José María Fernández Soria, "Influencias nacionales europeas en la política educativa española del siglo XX", Historia de la Educación, 24 (2005): 39-95.

${ }^{14}$ Jesús Gutiérrez Gassis, "El método de la pedagogía jurídica", Revista de ciencias jurídicas y sociales, 15 (1921): 402-423. 
Penal: Derecho Penal Especial y Criminología e Instituciones Penitenciarias, cursables desde tercero y cuarto respectivamente. Después vendrían las reformas de la Dictadura en 1924, 1926 y 1928. Fue con ocasión de esta última, como veremos, cuando se creó el Instituto de Estudios Penales.

En Valencia la asignatura de Derecho Penal fue impartida hasta la llegada de Enrique de Benito por José María Llopis Domínguez, Pedro Nolasco Mirasol y de la Cámara -este tan solo unos meses en 1896-, y Pascual Testor Pascual. Llopis fue el catedrático que conoció la separación de la asignatura de Derecho Mercantil, pues ocupó la cátedra en Valencia desde 1864. Pascual Testor, miembro del Partido Liberal en Valencia se dedicó más a la política -fue concejal del ayuntamiento de Valencia, diputado provincial, senador y gobernador civil en varias provincias- que a la cátedra ${ }^{15}$. Fue, como vamos a ver, el profesor Enrique de Benito quien introdujo los estudios de la Criminología en la Universidad de Valencia, especialmente con la creación del Instituto de Estudios Penales en 1928.

El profesor Enrique de Benito había nacido en Toledo en 1882. Estudió Derecho en Zaragoza y allí comenzó la docencia como profesor auxiliar numerario por oposición de Derecho Penal ${ }^{16}$. En diciembre de 1905 entró en el cuerpo de catedráticos numerarios por oposición al ganar la plaza de Derecho Penal en la Universidad de Santiago ${ }^{17}$. Un año después, en 1906, de Benito pasaba a la Universidad de Oviedo, siendo ya el encargado de pronunciar el discurso de apertura del curso académico 1906-1907 ${ }^{18}$. El atentado a los reyes Alfonso XIII y su esposa en la calle Mayor, obra de los anarquistas, fue el punto de partida para desarrollar un análisis criminológico sobre el anarquismo como fenómeno delictivo, sus factores individuales, físicos, antropo-

\footnotetext{
${ }^{15}$ Muy poca es la producción investigadora de Pascual Testor. Se conserva un discurso de apertura de 49 páginas sobre política universitaria. Discurso leído en la solemne inauguración de curso de la Universidad Literaria de Valencia por el Dr. D. Pascual Testor y Pascual, catedrático de la facultad de Derecho (1911-1912), (Valencia: Tip. Moderna, 1911).

${ }^{16}$ Sobre el profesor Enrique de Benito puede verse su perfil en el Diccionario de Catedráticos Españoles de Derecho (1847-1943). http://portal.uc3m.es/portal/page/portal/instituto_figuerola/programas/phu/diccionariodecatedraticos/lcatedraticos/ebenitollave (consultado el 24 de noviembre de 2019).

Su tesis doctoral nada tuvo que ver con el Derecho Penal pues versó sobre el Juicio crítico de las empresas políticas de Saavedra Fajardo y examen de su doctrina jurídica, tesis doctoral leída por D. Enrique de Benito el 13 de octubre de 1903, (Zaragoza, 1904).

${ }^{17}$ Para su oposición presentó ante el tribunal: "Concepto de Derecho penal y de su estudio: trabajo de investigación que somete al tribunal”, (1905), 112 páginas.

${ }^{18}$ Discurso leído en la solemne apertura del curso académico de 1906 a 1907 por D. Enrique De Benito, catedrático numerario de Derecho Penal, (Oviedo: Tip. Adolfo Brid, 1906), 71 págs.
} 
lógico y sociales, así como la prevención y represión de sus delitos, policía científica y régimen penitenciario para anarquistas. En Oviedo llevó a cabo una importante labor en la disciplina, con la creación de un Laboratorio y Museo de Criminología -lo había intentado en Zaragoza, sin éxito-, así como con la impartición de cursos extraordinarios de "Medicina Legal" o de "Policía Judicial Científica" -esta última una de sus preferencias en el ámbito de la Criminología-. Fruto de esos años fue su publicación La Cátedra, el Laboratorio y el Museo en la enseñanza del Derecho Penal, donde como él mismo decía, no hacía más que "exponer llanamente mi sistema de enseñanza del derecho penal" ${ }^{19}$. Abogaba por el estudio y la enseñanza "experimental" del derecho penal, algo que sin ser nuevo, no era todavía habitual en las universidades españolas, algo más en algunos países europeos y sudamericanos ${ }^{20}$. También publicó diversas obras relacionadas con el derecho Penal y con la Criminología, destacando obras como Policía Judicial Científica ${ }^{21}$ con la que continuaba profundizando en el desarrollo de la dactiloscopia -siguiendo los pasos de Federico Olóriz- o Delincuencia precoz, Individualización penal o El proceso de Guillermo II ante el Derecho Penal22.

En 1918, opositó a la cátedra de Derecho Penal de la Universidad Central, cátedra que no consiguió. Tras este intento, dos años después, de Benito solicitó el traslado por concurso a la Universidad de Valencia. En este caso, sí le fue concedido dicho traslado, tomando posesión de la cátedra de Derecho Penal de la Universidad de Valencia el 1 de febrero de $1921^{23}$. En ella desarrollaría su actividad docente hasta su fallecimiento, en 1930.

${ }^{19}$ Enrique de Benito y de la Llave, "El Derecho penal en la Cátedra”, Anales de Criminología de la Universidad de Oviedo (1907 a 1910), (Oviedo: Tip. de Flórez, 1912); La Cátedra, el Laboratorio y el Museo en la enseñanza del Derecho Penal. Tareas realizadas en la Universidad de Oviedo (años de 1905 y 1906), (Oviedo: Establecimiento tipográfico, 1907).

${ }^{20}$ Hace referencia a algunos museos y laboratorios criminológicos como el de Turín de Lombroso, el de Lyon o Toulouse, los laboratorios de psicología experimental en los Estados Unidos o el de la Universidad de La Habana, así como al recién creado en la escuela de Criminología de Madrid. Enrique de Benito y de la Llave, La Cátedra, el Laboratorio..., pp. 5, 12-13.

${ }^{21}$ Enrique de Benito y de la Llave, Policía judicial científica. Lecciones explicadas en la Universidad de Oviedo en los cursos especiales de 1913 a 1914 y 1914 a 1915. Manual para magistrados, jueces, abogados, peritos, agentes de policía y estudiantes de Derecho, (Madrid: Reus, 1915). Francisco Antón y Barberá, "Contribución española al auge en el uso de la dactiloscopia”, Gaceta Internacional de Ciencias Forenses, 27 (2018): 18-50, 34.

${ }^{22}$ Enrique de Benito y de la Llave, Delincuencia precoz, (Madrid: Arias, 1908); Individualización penal, (Madrid: Reus, 1916); El proceso de Guillermo II ante el Derecho Penal, (Madrid: Imp. de Ramona Velasco, 1919).

${ }^{23}$ Archivo de la Universidad de Valencia (en adelante AUV), Facultat de Dret, Registre i comunicacions, caixa 650. En septiembre de 1920 fallece Pascual Testor Pascual. 
El profesor de Benito desarrolló en la facultad de Derecho de Valencia una actividad tan intensa como la que había llevado a cabo en Oviedo. Desde el primer momento fue acogido por parte del resto de catedráticos de la facultad con verdadera querencia ${ }^{24}$. De Benito a su vez, mostró siempre una gran actividad académica que iba más allá de la puramente docente, ampliando estudios o impartiendo cursos y conferencias, como la que impartió recién incorporado a la Universidad de Valencia, en 1921, titulada "Las orientaciones modernas del Derecho Penal" y que fue publicada después en los Anales de la Universidad de Valencia ${ }^{25}$. También propuestas que hoy calificaríamos "de innovación docente", como visitas con sus estudiantes a diversos centros penitenciarios, como complemento práctico a su formación. Por ejemplo, en 1922 pasó un mes en la ciudad de Barcelona, junto con el profesor Luis Jordana de Pozas y un grupo de alumnos visitando instituciones oficiales y otras relacionadas con el mundo del Derecho y de la Ciencia Penal como, por ejemplo, la Prisión Provincial de Barcelona o el Tribunal para niños de la misma ciudad ${ }^{26}$. Igualmente, la Prisión Provincial de Valencia, la de

${ }^{24}$ Sobre la facultad de Derecho véase el completo estudio sobre la misma durante esos años de Jorge Correa Ballester y Francisco Javier Palao Gil, "La Facultad de Derecho de Valencia en el primer tercio del siglo XX (1900-1938)", en La enseñanza del Derecho en el siglo XX. Homenaje a Mariano Peset, ed. Adela Mora, (Madrid: Instituto Antonio de Nebrija de Estudios sobre la Universidad-Ed. Dykinson, 2004), 129-180.

${ }^{25}$ Enrique de Benito y de la Llave, "Las orientaciones modernas del Derecho Penal", Anales de la Universidad de Valencia, I-5 (1922), 380-418. En esta larga publicación dedica capítulos a la nueva Antropología criminal; la pena y las medidas de seguridad; los nuevos Códigos Penales; el Derecho Penal de menores; y a la Policía Judicial Científica. En 1923 pronunció otra conferencia en el Ateneo de Madrid, con el sugerente título El problema de la responsabilidad penal de los ministros. Conferencia pronunciada en el Ateneo de Madrid en la noche del 18 de febrero de 1923, (Madrid: Gráficas reunidas, 1923). También publicó otras conferencias como "Las novedades de la legislación penal extranjera (China, Italia, Rusia, 1919-1922), Anales de la Universidad de Valencia, IV-25-32 (1923), 161-178; "El derecho Penal Internacional y la Sociedad de Naciones", Anales de la Universidad de Valencia, VI-41-48 (1925), 96-110.

${ }^{26}$ Las instituciones que visitaron y de las cuales hicieron constar el exquisito recibimiento del que disfrutaron fueron: Grupo de protección a la Infancia (director, José María Folch); Junta Provincial de Protección a la Infancia (J. Rosa); Facultad de Derecho de la Universidad de Barcelona; Escuela de funcionarios de Administración Local (Director, Isidro Lloret); Mancomunidad de Cataluña; Casa de la Caridad de Barcelona (Secretario, Luis Nogueras); Diputación Provincial de Barcelona; Caja de Pensiones para la vejez y ahorro de Barcelona (Director, Francisco Moragas); Comité de la exposición Internacional de Industrias y general española (Secretario, Sans y Buigas); Ayuntamiento de Barcelona (alcalde); Prisión Provincial de Barcelona; Tribunal para niños de Barcelona (Ramón Albó y sr. Bassols). AUV, Facultat de Dret, Registre i comunicacions, caixa 666. 
Alicante o el Penal de Chinchilla fueron algunos de los centros penitenciarios que el profesor de Valencia visitó con sus estudiantes ${ }^{27}$.

Como docente, pues, Enrique de Benito fue un ejemplo paradigmático de uno de los perfiles de profesor que más se desarrolló durante los treinta primeros años del siglo XX. Tras el desastre del 98 se produjo lo que podríamos llamar el despertar de un prolongado letargo. El regeneracionismo, como en la política, la economía o en el seno de la intelectualidad, también hizo presencia en la Universidad. Profesores que no solo se dedicarían a la docencia, como habían predominado, con honrosas excepciones, hasta entonces, sino que añadirían a esa labor de enseñantes un destacable bagaje que hoy llamaríamos investigador ${ }^{28}$. Sus viajes de estudio al extranjero para ampliar y conocer las nuevas corrientes científicas fueron continuos, así como su participación en congresos internacionales. De Benito demostró en todas sus publicaciones un amplio conocimiento de los autores y corrientes extranjeras, no solo criminológicas sino también de otras materias como el derecho procesal, administrativo o constitucional. En sus obras encontramos citados a autores como Von Listz, Lombroso, Ferri, Garofalo, Ferriani, Alongi, Mezger o Benedikt. Coinciden sus planteamientos con muchos de los presupuestos de la Escuela Italiana, con la que no deja de discrepar en algunas cuestiones -como la clasificación de los delincuentes de Lombroso-. Se mostró siempre partidario de un Derecho penal dinámico, más amplio y experimental, no anquilosado en la legislación positiva. Además, para él era necesario -y su trayectoria así lo demostraba- examinar las leyes penales a la luz de la legislación comparada, europea y americana, y no solo con "mezquinos análisis exegéticos, sino que ha de comprender la tendencia filosófica, el alcance y fundamentos sociológicos y las aplicaciones antropológicas de cada precepto"29.

Su implicación por el Derecho Penal y la Criminología traspasó lo puramente académico involucrándose en instituciones civiles y proyectos in-

${ }^{27}$ En abril de 1925 de Benito daba cuenta de su visita con estudiantes al penal de Chinchilla, y la buena acogida que le dispensó su director. AUV, Facultat de Dret, Actas de la Facultad de Derecho (10/2/1925-2/12/1932), 30 de abril de 1925. En mayo de 1929 fue autorizado para visitar las cárceles de Valencia y Alicante por el director General de Prisiones, AUV, Facultat de Dret, Registre de comunicacions. Caixa 651.

${ }^{28}$ Octavio Ruiz-Manjón, "Los intelectuales en la política española del primer tercio del siglo XX", en Modernizar España. Proyectos de reforma y apertura internacional (1898-1914), eds. Guadalupe Gómez-Ferrer y Raquel Sánchez, (Madrid: Biblioteca Nueva, 2007), 61-77. En la misma obra colectiva y en el mismo sentido, Antonio Niño Rodríguez, "El protagonismo de los intelectuales en los proyectos de reforma educativa y modernización cultural", 199-229.

${ }^{29}$ Enrique de Benito y de la Llave, La Cátedra, el Laboratorio..., p. 8. 
ternacionales que buscaban las mejores respuestas ante los problemas que podía plantear la criminalidad en los tiempos presentes. En Oviedo, de Benito fue miembro de la Junta de Patronato de la prisión de aquella ciudad y de la Comisión de Libertad Condicional. Representó a la Universidad de Oviedo en el Congreso Internacional contra la Trata de Blancas celebrado en 1910. Participó en congresos como el Congreso Nacional de Educación Protectora de la Infancia abandonada, viciosa y delincuente en 1908 o el Congreso Penitenciario celebrado en Londres de 1925.

En su vida personal, Enrique de Benito fue un hombre católico practicante cuyo único coqueteo formal con la política fue su participación en la constitución del Partido Social Popular, en 1922, de muy corta vida -la versión española del Partido Popular Italiano de Sturzo-. En Valencia, fue miembro y consejero de la Asociación y cátedra de Luis Vives y de la academia valencianista del Centro de Estudios Mercantiles ${ }^{30}$. Aunque muy reconocido por sus compañeros de cátedra, sin embargo, sus enfrentamientos con los estudiantes en las huelgas y disturbios de 1929-30, para muchos fueron la causa indirecta de su repentino fallecimiento. ${ }^{31}$

En 1925, becado y autorizado por la Universidad viajaba a Francia y Bélgica con el fin de estudiar algunas instituciones penitenciarias de dicho país. En la capital francesa, así como en Bruselas, entabló contactos con miembros de las principales instituciones penitenciarias y jurídicas de ambas ciudades ${ }^{32}$. Fruto de este viaje de estudios fue su publicación La actual organización de la lucha contra el delito en Francia, publicada en 1926. Dos años después, en 1927, de Benito solicitaba al claustro de profesores de la facultad permiso para pasar un año por distintos países sudamericanos con

\footnotetext{
${ }^{30}$ En ella se integraba el sector valencianista y católico en la órbita de catedráticos como Rafael Rodríguez de Cepeda, Luis Gestoso, el marqués de Lozoya o Mariano Puigdollers. María Fernanda Mancebo, La Universidad de Valencia. De la Monarquía a la República (1919-1939), (Valencia: Universitat de València, Instituto de Cultura Juan Gil-Albert, 1994), 72-74.

${ }^{31}$ María Fernanda Mancebo, La Universidad de Valencia..., p. 335.

${ }^{32}$ Algunas de las personalidades con las que entabló contacto fueron H. Bertheleny, miembro del Instituto de Francia y decano de la facultad de Derecho de París; con L. Hugueney y H. Domnedieu de Valves, profesores de derecho Penal de la expresada facultad; Aubry y $\mathrm{H}$. Rollet, presidente y juez del tribunal de Sena; Bayle, director del Instituto Judicial de París; Balthazard, director del Instituto Médico Legal de París; P. Kahn y D. Mercier, abogados del Tribunal de Apelación de París; y Cazave, Jefe de Despacho del Director General de Prisiones. Finalizado ese mes, alargó su estancia en Bélgica donde se reunió con el Director del Servicio de Antropología Penitenciaria, el Juez de niños de Bruselas y el Director de la Asistencia Pública de aquella ciudad. AUV, Facultat de Dret, Actas de la Facultad de Derecho (10/2/19252/12/1932), 23 de octubre de 1925.
} 
el fin de impartir diferentes cursos y ampliar estudios. Primero en la Universidad Libre de Lima donde tenía intención de ofrecer un curso de Estudios Superiores de Derecho Penal y dos cursos breves de Ciencia Penitenciaria y Derecho Penal de Menores respectivamente, que orientasen a aquel país en la reforma de sus prisiones y en la creación allí de Tribunales para niños. Después, aprovecharía "para ampliar estudios de Derecho Penal en alguna de aquellas Repúblicas", por lo que su estancia se alargaría un año. A tal efecto, el claustro de profesores apoyó la solicitud que hizo de Benito a la Junta para la Ampliación de Estudios para que fuera considerado pensionado, aunque sin retribución económica alguna ${ }^{33}$. Tras el regreso de su largo viaje de estudios por Hispanoamérica fue cuando por fin un proyecto que se había iniciado en 1926 se haría realidad.

\section{El Instituto de Estudios Penales}

El proyecto que perseguía Enrique de Benito fue propuesto a la Junta de catedráticos de la facultad de Derecho a finales de 1925. Dada su trayectoria, a nadie podía sorprenderle ya la intención del profesor de crear un Instituto de Estudios Penales. Sin oposición alguna a dicho proyecto, unos meses después, de Benito presentaba el borrador de los "Estatutos para un Instituto de Estudios Penales dependiente de la Universidad de Valencia" ${ }^{34}$. Se trataba de una regulación somera dividida en diez artículos, por el que se creaba un Instituto dependiente de la facultad de Derecho que tendría la consideración de "permanente". En el Instituto se impartirían un "conjunto de enseñanzas teóricas y prácticas referentes al Derecho Penal y a sus disciplinas auxiliares y afines para ampliación del programa oficial de la mencionada asignatura y con el propósito, además, de que puedan solicitar y obtener, quienes las hayan recibido asiduamente, un certificado o diploma que lo acredite". La sede física del Instituto se ubicaba en las propias dependencias de la facultad, la cual tendría sobre el Instituto el patronato, inspección y dirección del mismo. El Instituto tendría, a su vez, su propia Junta de profesores, la cual elegiría a un director, necesariamente un catedrático del Claustro, y a un secretario.

${ }^{33}$ AUV, Facultat de Dret, Actas de la Facultad de Derecho (10/2/1925-2/12/1932), 9 de abril de 1927. Así fue reconocido a través de la Real Orden de 7 de junio de 1927. Durante su ausencia, se haría cargo de la enseñanza de Derecho penal su ayudante, el profesor Ángel Moliner Gimeno.

${ }^{34}$ AUV, Facultat de Dret, Actas de la Facultad de Derecho (10/2/1925-2/12/1932), 11 de mayo de 1926. 
El curso quedaba diseñado para que fuera impartido a partir de enero o febrero hasta mayo y cuyas asignaturas, profesores, horarios y espacios serían organizados por la propia Junta de profesores del Instituto. Los requisitos para poder matricularse tenían que ser: haber aprobado la asignatura de Derecho Penal, en cualquier Universidad, o la asignatura de Medicina Legal, para los estudiantes de Medicina. También podían inscribirse en el Instituto, las personas que desempeñaran cargo o profesión relacionados con la Administración de Justicia y las que hubieran acreditado hallarse preparadas para tomar parte en los concursos u oposiciones que hubieran de anunciarse para proveerlos. Finalizado el curso con éxito, tras las pruebas que se hicieran en su caso, a los alumnos se les libraría el correspondiente certificado o diploma de haber cursado las enseñanzas en las que se hubieran matriculado. Estos certificados o diplomas serían expedidos por la facultad de Derecho y tendrían la validez que el gobierno les quisiera conceder.

Cuando de Benito presentó su proyecto lo hizo con vocación de continuidad, siguiendo el modelo del Instituto de Idiomas, creado en 1918, y que tan bien estaba funcionando ${ }^{35}$. Para ello debía autofinanciarse en la medida de lo posible a través de los recursos siguientes: el producto de las inscripciones de matrícula y exámenes; subvenciones concedidas por el gobierno; el beneficio que se pudiera obtener de sus publicaciones; $y$ donativos y subvenciones tanto de entidades académicas como no académicas, así como los ingresos que procedieran de particulares que quisieran contribuir al sostenimiento del Instituto. Gestión económica que quedaba bajo la supervisión de la Junta de profesores del Instituto, ante la Junta de la facultad y de la Junta económica de la Universidad. Terminaban los Estatutos con la prevención de su posible disolución y, en ese caso, destino de sus recursos sobrantes a la facultad de Derecho.

En cuanto a la enseñanza del Instituto poco o nada decía el reglamento. Ni asignaturas, designación de docentes, plazas que se ofertaban..., nada. En la sesión en la que se presentó el proyecto no hubo ninguna opinión en contra, lo que hacía pensar en una inminente instalación del Instituto. Sin embargo, no habrá noticia alguna del Instituto hasta dos años después. Entre medias, el viaje por un año del profesor de Benito a Sudamérica para ampliar conocimientos y a su vez impartir cursos y conferencias sobre cuestiones penitenciarias y criminológicas. Es a su vuelta, a comienzos del curso 192829, cuando el Instituto comienza, como vamos a ver, su brevísima andadura.

${ }^{35}$ Sobre el Instituto de Idiomas de la Universidad de Valencia, María Fernanda Mancebo, La Universidad de Valencia..., 66-72. 
En los momentos más difíciles de la dictadura de Primo de Rivera, su ministro de instrucción pública propondría una reforma universitaria que le complicaría aún más la permanencia al dictador. En 1928, Eduardo Callejo de la Cuesta presentaba al directorio civil una reforma que se hizo efectiva a través del Decreto-Ley de 19 de mayo de aquel año ${ }^{36}$. Un decreto que, aunque hablaba de autonomía o de libertad de cátedra entre otras cosas, lo bien cierto es que éstas realmente no estaban por ningún lado. El plan de estudios dividía las enseñanzas en tres tipos: Cursos A, que eran las enseñanzas obligatorias de cada una de los estudios universitarios; Cursos B, o profesionales, tanto de enseñanzas que pudieran adiestrar para el ejercicio profesional o ser básicas para el mismo, como preparaciones concretas de ingreso en cuerpos del estado por oposición; y Cursos C, para los estudios del doctorado. Pues bien, fue con la aplicación de esta reforma cuando se instaló el Instituto adoptando la forma de Curso $\mathrm{B}^{37}$. Ese mismo año se iba a aprobar el nuevo Código Penal que se estaba elaborando y que desde luego asumía los planteamientos de la Escuela Positiva. Tenía mucho sentido la puesta en marcha del Instituto como complemento a la enseñanza de los estudiantes de las facultades de Derecho ${ }^{38}$.

Siguiendo lo que se establecía en el Decreto-Ley, las enseñanzas de este tipo, y en este caso las del Instituto, debían ser teórico-prácticas. Desde la facultad se tenía que enviar al Ministerio la propuesta de asignaturas adicionales al plan de licenciatura del Real Decreto, así como la propuesta de cursos B o profesionales. El propio real decreto señalaba lo interesante de este tipo de cursos dirigidos fundamentalmente a la preparación de oposiciones a profesiones especializadas o a la profesión libre. Estos cursos, de esta manera, se concebían como una formación complementaria que podía organizarse por las tardes, impartida por los catedráticos de la facultad correspondiente para conservar el tono científico exigido. Se aconsejaba también utilizar los servicios del profesorado auxiliar o ayudante para la parte de repetición, con lo que ese personal podría tener un justificado aumento

${ }^{36}$ Sobre la aplicación de la reforma de Callejo en la Universidad de Valencia, María Fernanda Mancebo, La Universidad de Valencia..., 49-98. Sobre el ministro, Antonio Calonge Velázquez, "Eduardo Callejo de la Cuesta un ministro primorriverista", Revista Aequitas, 10 (2017): 137-173.

${ }^{37}$ El Plan del ministro Callejo se discute en la Junta de profesores de la facultad de Derecho durante varias sesiones. AUV, Facultat de Dret, Actas de la Facultad de Derecho (10/2/19252/12/1932), 1, 2, 4, 6 y 20 de junio de 1928.

${ }^{38}$ Gabriela Cobo del Rosal, "El proceso de elaboración del Código Penal de 1928”, Anuario de Historia del Derecho, 82, (2012): 562-602. 
de remuneración, así como con la colaboración de especialistas de cada preparación profesional que enseñarían su técnica especializada. Esta preparación para oposiciones a profesiones especializadas o para la profesión libre podría establecerse para alumnos de los dos últimos años de cada facultad y para licenciados, sin perjuicio de simultanear enseñanzas básicas de estos cursos B en todos los años, procurando armonizarlas con las preparaciones diferenciadas. Por otro lado, estos cursos B podían formar parte de los cursos de doctorado -cursos C-. Para la facultad de Derecho se diseñaron tres planes de doctorado: Plan de Derecho Civil; Plan de Derecho Público; Plan de Ciencias Sociales. Era en estos dos últimos planes, en los que el estudiante podía, en sustitución del curso o cursos tipo B, optar por enseñanzas del Instituto de Estudios Penales.

Así pues, el Instituto de Estudios Penales creado, entre otras cosas, por el interés que representaba para la facultad de Derecho el nuevo Código Penal -el de 1928- y ajustándose a la reforma de Callejo se diseñó con las siguientes enseñanzas: Jurisprudencia Criminal; Derecho Penal de los Menores; Ciencia y Legislación Penitenciaria; Psicología Criminal; Medicina Legal; y Policía Científica ${ }^{39}$. El plan de estudios fue proyectado en relación al profesorado con el que se contaba. La Antropología criminal, disciplina fundamental en la ciencia criminológica y presente en los planes de estudio de 1903 y 1917 de la Escuela de Criminología de Madrid, no se impartió en el Instituto por no contar con especialistas en la materia. Sí, en cambio, el Derecho Penal de Menores, enseñanza en la que el propio de Benito tenía amplios conocimientos, estando en la órbita de otros catedráticos como Inocencio Jiménez, José Guallart o Federico Castejón. Medicina Legal era una novedad en el Instituto, respecto de la Escuela de Criminología. En este caso se trataba de aprovechar las enseñanzas de Juan Peset que había convertido la cátedra valenciana "en uno de los principales centros de la disciplina" ${ }^{40}$. Para Legislación Penitenciaria y Psicología criminal se buscó especialistas en ambos campos: el director de la Prisión de Valencia y el doctor GómezFerrer. Por último, Policía Científica y todo lo referente a la dactiloscopia, era la especialidad del profesor de Benito. Esta asignatura, que como tal no vemos en los planes de la Escuela de Criminología, sin embargo, no podía faltar

\footnotetext{
${ }^{39}$ Esta última enseñanza sería complementada por la que había creado la facultad de Filosofía y Letras relativa a "Análisis y reconocimiento pericial de la escritura" o "Peritaje judicial de escrituras" y constituyó, juntamente con ella, un solo curso dividido en dos partes.

${ }^{40}$ José Ma López Piñero, "La facultad de Medicina de Valencia", en Historia de la Universidad de Valencia. Volumen III: La Universidad liberal (Siglos XIX y XX), coord. Mariano Peset, (Valencia: Universitat de València, 2000), 178-208, p. 207.
} 
en su Instituto. En definitiva, se trataba de una Escuela hecha a la medida del estado de la ciencia criminológica en Valencia.

En cuanto a su organización, cada asignatura tenía una duración de una hora semanal. El periodo de estos cursos se preveía de tres meses y sería necesario asistir, al menos, a tres de los cursos señalados a elección del estudiante para poder obtener el oportuno certificado de estudios. Como ya he señalado, podían ser admitidos al Instituto, los alumnos de la facultad de Derecho que hubieran aprobado el curso de Derecho Penal de la licenciatura o el análogo del Doctorado, así como los licenciados o doctores en Derecho y Medicina y las personas que sin reunir estos requisitos ejercieran empleos o desempeñaran servicios para los cuales fuera de interés seguir estos cursos, a juicio de la Junta de profesores de la facultad de Derecho ${ }^{41}$. La dirección del Instituto finalmente fue confiada al catedrático de Derecho Penal y artífice del mismo, el profesor Enrique de Benito.

Después de dos años desde su presentación, el 14 de diciembre de 1928 se aprobaba el plan de estudios del Instituto de Estudios Penales para el curso 1928-29. El curso trimestral, tal y como estaba previsto en el Real Decreto, comenzó en febrero de 1929 y acabó a finales de mayo. Un informe del propio De Benito nos da los detalles de aquel primer curso que impartió el Instituto de Estudios Penales.

Los cursos B constitutivos del mencionado Instituto han funcionado con total normalidad. Era de temer que tratándose de un organismo nuevo comprensivo de estudios que requieren una nueva preparación especial, que no ocurriese así y que alguna, al menos, de las enseñanzas que han constituido el cuadro de las del Instituto no pudieran darse por falta de matrícula. Pero afortunadamente, no ha sucedido así: en todos los cursos ha habido matrícula de modo que la facultad de Derecho de la Universidad de Valencia ha cumplido exactamente cuánto se propuso y anunció al público al crear los cursos B constitutivos del Instituto de Estudios Penales ${ }^{42}$.

En el informe se señalaba, además, que las enseñanzas, sin dejar de ser especulativas, habían tenido el exigido carácter práctico y profesional, con lecciones semanales teórico-prácticas y trabajos de experimentación. Los programas de cada una de las asignaturas se habían cumplido en su totalidad, programas redactados por cada uno de sus profesores con la debida antelación. Los cursos, aunque trimestrales habían acabado alargándose casi un

\footnotetext{
${ }^{41}$ AUV, Facultat de Dret, Actas de la Facultad de Derecho (10/2/1925-2/12/1932), Actas del 1, 2, 4, 6 y 20 de junio de 1928.

${ }^{42}$ AUV, Facultat de Dret, Registre de comunicacions. Caixa 670. Informe fechado el 14 de junio de 1929.
} 
Junta de Profesores del Instituto de Estudios Penales (1929-1930)

\begin{tabular}{|l|l|l|}
\hline \multicolumn{1}{|c|}{ Profesor IEP } & \multicolumn{1}{|c|}{ Facultad/Institución } & \multicolumn{1}{c|}{ Asignatura IEP } \\
\hline Luis Gonzalvo Paris ${ }^{\mathrm{a}}$ & $\begin{array}{l}\text { Filosofía y Letras y } \\
\text { Archivero (catedrático) }\end{array}$ & $\begin{array}{l}\text { Peritaje judicial de } \\
\text { escrituras }\end{array}$ \\
\hline $\begin{array}{l}\text { Juan Bautista Peset } \\
\text { Aleixandre }\end{array}$ & Medicina (catedrático) & Medicina Legal \\
\hline Pedro Gómez-Ferrer Martíc $^{\text {b }}$ & $\begin{array}{l}\text { Laboratorio de Psicología } \\
\text { Experimental y Orientación } \\
\text { Profesional del Reformatorio } \\
\text { de Menores de Godella }\end{array}$ & Psicología criminal \\
\hline Vicente Rodríguez Ferrer ${ }^{\mathrm{d}}$ & $\begin{array}{l}\text { Prisión Provincial de } \\
\text { Hombres de Valencia }\end{array}$ & $\begin{array}{l}\text { Ciencia y Legislación } \\
\text { penitenciaria }\end{array}$ \\
\hline Antonio Alarcón & Derecho (profesor auxiliar) & Jurisprudencia criminal \\
\hline Ángel Moliner & Abogado & Derecho Penal de menores \\
\hline Enrique de Benito & Derecho (catedrático) & Policía científica \\
\hline
\end{tabular}

a Sobre Luis Gonzalvo Paris, J. Emili Aura Tortosa, "La creació del Laboratori d'Arqueologia de la Universitat de València: Entre l'Escola Superior de Diplomática i «por la ciencia hacia Dios» (1921-1940)", en Arqueologia en blanc i negre: la labor del SIP,1927-1950, coords. Helena Bonet Rosado, María Jesús de Pedro Michó, Ángel Sánchez Molina i Carles Ferrer García, (València: Diputació de València, 2006), 33-46.

b Sobre el malogrado rector Peset, fusilado en 1941 por el régimen franquista, VV. AA., Proceso a Juan Peset Aleixandre (Valencia: Universitat de València, 2001); Marc Baldó, María Fernanda Mancebo, "Juan Peset Aleixandre: científico, político, ciudadano", en Republicanos en la memoria: Azaña y los suyos, coord. Ángeles Egido, (León: Eneida, 2006), 199-210.

${ }^{\mathrm{c}}$ El doctor Gómez-Ferrer había participado un año antes, en 1928, en la película València, protectora de l'infància, de Maximiliano Thous. Se trataba de un documental que ilustraba el trabajo que hacían algunas instituciones valencianas en favor de la infancia en el ámbito de la sanidad, educativa o judicial. El documental puede verse en: http://www.restauracionesfilmoteca.com/cine-valenciano/no-ficcion/valencia-protectora-de-la-infancia/

${ }^{\mathrm{d}}$ Vicente Rodríguez Ferrer fue autor de un Manual de identificación judicial (dactiloscopia, filiación descriptiva, fotografía): reglas prácticas, (Madrid, 1914).

mes más porque así lo habían solicitado profesores y alumnos, a fin de poder ultimar los respectivos programas. Todos se habían impartido en la facultad de Derecho menos los de Psicología Criminal y Medicina Legal que se dieron en la de Medicina, por los materiales para trabajos prácticos que se requerían en estos dos cursos. Por desgracia, no contamos con dichos programas, por lo que desconocemos el contenido de las enseñanzas de estas asignaturas.

Sí que conocemos los profesores que las impartieron. El cuadro de profesores de aquel primer curso del Instituto de Estudios Penales había sido nombrado por la propia facultad, a propuesta del profesor de Benito. A 
ellos se refería en su informe dedicando palabras de elogio por la generosidad y el buen hacer de todos ellos ${ }^{43}$.

El Instituto tuvo aquel primer curso nueve alumnos y en relación a ellos de Benito hacía constar que todos habían asistido con asiduidad y habían demostrado gran aplicación. Destacaba que algunos de los matriculados eran "elementos ajenos a la clase escolar universitaria, pero que por sus empleos desempeñan misiones de policía judicial para las que son útiles ciertos conocimientos criminológicos", entre ellos oficiales de la Guardia Civil y del cuerpo de Prisiones. Por otra parte, tan solo cuatro de ellos eran estudiantes de Derecho, entre los que se encontraba la única mujer: Luz Le Boucher.

En el curso siguiente, 1929-1930, las asignaturas volvieron a ser las mismas, aunque en este caso no tenemos constancia de quiénes fueron los profesores que las impartieron. En cuanto al número de alumnos, veinte alumnos solicitaron en diciembre de 1929 cursar algunas de las asignaturas del Instituto, que en este segundo curso se desarrolló entre enero y mayo de 1930. En este caso, además de las materias cursadas, se recogían datos personales como la edad o su lugar de origen ${ }^{44}$. Más de la mitad eran estudiantes de Derecho -entre ellos los futuros profesores Diego Sevilla Andrés y Felipe Garín Ortiz de Taranco-; un opositor a la policía nacional; un empleado de prisiones de San Miguel de los Reyes; cuatro religiosos capuchinos dedicados a la educación de jóvenes en reformatorios e instituciones similares; y una mujer, natural de Cuenca y agente de vigilancia.

${ }^{43}$ En el informe se señalaba que la facultad había resuelto poner a disposición del Instituto lo recaudado por matrículas -15 pesetas por asignatura- y derechos de certificados -50 pesetas-, y satisfechos algunos gastos de material, el remanente de setecientas pesetas podía distribuirse entre los profesores como retribución de sus cursos. Algunos de ellos renunciaron a dicha gratificación y dejaron lo que les correspondía en beneficio del naciente Instituto. AUV, Facultat de Dret, Registre de comunicacions. Caixa 670. Efectivamente, el 7 de octubre de 1929 se leyeron las cuentas del Instituto de Estudios Penales correspondientes al año 1929 presentadas por su director. En ellas se hacía constar que los honorarios devengados por el director, profesor De Benito y de los profesores del mismo, Peset, Gonzalvo y Gómez Ferrer como dotación de sus cursos respectivos y que sumaban un total de 400 pesetas, dejaron de ser percibidos voluntariamente, quienes hicieron donación de sus haberes para las necesidades del Instituto. La Junta acordó aprobar las cuentas, expresar su beneplácito por la labor realizada y que constara en acta su felicitación y gratitud al profesorado del Instituto y muy singularmente a su director sr. De Benito. Igualmente acordó proponer a la Junta de gobierno de la Universidad que consignara en los presupuestos de la misma una subvención, como mínimo de 500 pesetas, para contribuir al sostenimiento del Instituto. AUV, Facultat de Dret, Actas de la Facultad de Derecho (10/2/1925-2/12/1932), Acta del 7 de octubre de 1928.

${ }^{44}$ AUV, Facultat de Dret, Caixa 699. 
El nuevo curso del Instituto comenzó en un momento crítico para la monarquía de Alfonso XIII. El 30 de enero de 1930 dimitía el dictador Miguel Primo de Rivera. Era sustituido por otro general, Dámaso Berenguer que, ni convocaría a las Cortes suspendidas en 1923, ni volvería a poner en vigor la Constitución de 1876. El fin de la dictadura, lo que sí que provocó a nivel universitario es que volviera a decretarse apresuradamente un nuevo plan de estudios a través del Real Decreto de 25 de septiembre de ese mismo año para el nuevo curso que iba a comenzar. El 3 de octubre de 1930 se daba cuenta del nuevo plan presentado por el valenciano Elías Tormo el cual, en principio, no iba a afectar a la permanencia o no del Instituto. Efectivamente, el 16 de octubre se hacía mención al Instituto de Estudios Penales, "que viene funcionando con éxito satisfactorio", por lo que continuaría con su enseñanza, arreglándose a la nueva estructuración ${ }^{45}$. Pero todo se iba a precipitar de una manera absolutamente imprevista para el Instituto y su director. El 27 de octubre fallecía repentinamente el profesor De Benito, a los 48 años de edad. Los periódicos daban cuenta de cómo había sido el triste suceso.

Esta mañana a las nueve, cuando iba a entrar en el salón de profesores de esta Universidad el de Derecho penal, D. Enrique de Benito, sufrió un ataque cerebral y se apoyó en el quicio de la puerta. Asistido por el portero mayor. Sr. Herrero, y el profesor auxiliar Sr. Cortés, que salió rápidamente de dicha sala, fue entrado en ella, y colocado en un sillón, y mientras el Sr. Herrero le preguntaba si se le había pasado inclinó el Sr. De Benito la cabeza y falleció. El cadáver ha sido colocado en la capilla privada de la Universidad. El rector, Sr. Zumalacárregui, con el Claustro, ha adoptado las disposiciones propias del caso. Entre ellas, telegrafiar a los familiares del Sr. De Benito, residentes en Zaragoza y que, según nuestras noticias, han salido ya para nuestra ciudad. El cadáver será trasladado a la ciudad nombrada ${ }^{46}$.

Durante todo el día 28 fue velado el cuerpo del profesor de Benito en la capilla de la Universidad "por el rector y sus compañeros, el director y la redacción del Diario Valenciano y comisiones de estudiantes, que se fueron substituyendo". Durante el día siguiente se hicieron misas "cada media hora" y se suspendieron las clases. El cadáver fue embalsamado, precisamente, por uno de los profesores del Instituto, el doctor Juan Bautista Peset. Antes de partir hacia Zaragoza, donde fue enterrado, fue llevado a hombros por compañeros y familiares dando una vuelta por el claustro central de la Universidad ${ }^{47}$.

${ }^{45}$ AUV, Facultat de Dret, Acta de la Facultad de Derecho (10/2/1925-2/12/1932), Acta del 16 de octubre de 1930

${ }^{46} A B C$ (Madrid), martes 28 de octubre de 1930, p.44.

${ }^{47}$ ABC (Madrid), martes 29 de octubre de 1930, p. 32. Presidieron los actos el rector de la Universidad Zumalacárregui, el representante del gobernador civil, Sr. Afán de Ribera; el del 
El 4 de noviembre se informaba del fallecimiento al resto del claustro de profesores. Ese mismo día se acordaba la suspensión del funcionamiento del Instituto hasta que se incorporara el nuevo catedrático de Derecho Penal. Por R. O. de 12 de noviembre de 1930 fue nombrado con carácter interino José Arturo Rodríguez Muñoz, excedente en expectación de destino, como catedrático de Derecho Penal de la facultad de Derecho de Valencia ${ }^{48}$. Pero la llegada a Valencia de Rodríguez Muñoz no trajo consigo el restablecimiento del Instituto, tal y como se había manifestado en la Junta de facultad, quien sabe si llevados por las buenas intenciones del momento. De Benito había fallecido y con él, el Instituto de Estudios Penales de Valencia.

\section{Consideraciones finales}

Tras la muerte del catedrático de Benito, el Instituto no se restableció. Tampoco en los años siguientes de la República, ni durante los largos años del franquismo. La Criminología como disciplina especializada desapareció de la facultad. Algún curso especial, pero poco más. Los planes no sufrieron cambios tan intensos como había sucedido en los años previos a la Guerra civil. A partir de los años cincuenta más estabilidad: el Plan de 1953 primero, y después el plan de 1965 del ministro Lora-Tamayo que llegará hasta bien entrada la democracia. Fue precisamente en los años inmediatamente posteriores a su publicación, cuando volvió a resurgir la experiencia de los años veinte ${ }^{49}$.

Siendo catedrático de Derecho penal el profesor Juan Córdoba Roda, se volvió a proponer la creación de un Instituto de Criminología ${ }^{50}$. En 1968 se presentaba al Consejo Nacional de Educación el reglamento para el nuevo Instituto de Criminología de Valencia. Por una Orden Ministerial de 17 de agosto de 1968 se aprobaba su creación. El Instituto fue inaugurado solemnemente el 19 de febrero de 1969. Pocos días después se hacía oficial el funcionamiento de este nuevo Instituto con la celebración de unas Jornadas

arzobispo, deán, Sr. Payá, el presidente de la Audiencia, Sr. Suárez, el presidente de la Diputación, Sr. Serrano, y por la familia, el general Sr. de. Figueras.

${ }^{48}$ AUV, Facultat de Dret, Acta de la Facultad de Derecho (10/2/1925-2/12/1932), Acta del 20 de noviembre de 1930.

${ }^{49}$ AUV, Facultat de Dret, Actas de Juntas de Facultad, 10 de noviembre de 1967-22 de noviembre de 1969. Acta del 10 de julio de 1968, fol. 25v.

${ }^{50}$ Mariano Peset, "Rebelión en la Universidad”, en La Facultad de Derecho de Valencia, 14991975, coords. Mariano Peset y Jorge Correa, (Valencia: Universitat de València, 2018), 388. 
Hispano-Francesas de Criminología con la presencia de Jean Pinatel, secretario general de la Sociedad Internacional de Criminología, Pierre Bouzat, secretario general de la Asociación Internacional de Derecho Penal y Octavio Pérez-Vitoria, director del Instituto de Criminología de Barcelona ${ }^{51}$. La Criminología volvía a Valencia, después de una larga travesía en el desierto.

\section{Bibliografía}

ANTÓN y BARBERÁ, Francisco, "Contribución española al auge en el uso de la dactiloscopia”, Gaceta Internacional de Ciencias Forenses, 27 (2018), 18-50, 34.

Antón OneCA, José, “Don Rafael Salillas", Anuario de Derecho Penaly Ciencias Penales, 27, fasc. II (1974): 205-220.

Aura Tortosa, J. Emili, "La creació del Laboratori d'Arqueologia de la Universitat de València: Entre l'Escola Superior de Diplomática i «por la ciencia hacia Dios» (1921-1940)", en Arqueologia en blanc i negre: la labor del SIP,1927-1950, coords. Helena Bonet Rosado, María Jesús de Pedro Michó, Ángel Sánchez Molina i Carles Ferrer García, (València: Diputació de València, 2006), 33-46.

Baldó, Marc; María Fernanda Mancebo, "Juan Peset Aleixandre: científico, político, ciudadano", en Republicanos en la memoria: Azaña y los suyos, coord. Ángeles Egido, (León: Eneida, 2006), 199-210.

BENITO Y DE LA LLAVE, ENRIQUE DE, Juicio crítico de las empresas políticas de Saavedra Fajardo y examen de su doctrina jurídica, tesis doctoral leída por D. Enrique de Benito el 13 de octubre de 1903, (Zaragoza, 1904).

BENITO Y DE LA LLAVE, ENRIQUE DE, Discurso leído en la solemne apertura del curso académico de 1906 a 1907 por D. Enrique De Benito, catedrático numerario de Derecho Penal, (Oviedo: Tip. Adolfo Brid, 1906).

BENITO Y DE LA LLAVE, ENRIQUE DE, La Cátedra, el Laboratorio y el Museo en la enseñanza del Derecho Penal. Tareas realizadas en la Universidad de Oviedo (años de 1905y 1906), (Oviedo: Establecimiento tipográfico, 1907).

Benito y De la Llave, EnRIQue DE, Delincuencia precoz, (Madrid: Arias, 1908).

Benito y DE la Llave, EnRIQue DE, "El Derecho penal en la Cátedra", Anales de Criminología de la Universidad de Oviedo (1907 a 1910), (Oviedo: Tip. de Flórez, 1912).

\footnotetext{
${ }^{51}$ La Vanguardia, 2 de marzo de 1969, p. 8.
} 
Benito y De la Llave, EnRiQue De, Policía judicial científica. Lecciones explicadas en la Universidad de Oviedo en los cursos especiales de 1913 a 1914 y 1914 a 1915. Manual para magistrados, jueces, abogados, peritos, agentes de policía y estudiantes de Derecho, (Madrid: Reus, 1915).

BENITO Y DE LA LLAVE, ENRIQUE DE, Individualización penal, (Madrid: Reus, 1916).

Benito y DE la Llave, EnRIQue DE, El proceso de Guillermo II ante el Derecho Penal, (Madrid: Imp. de Ramona Velasco, 1919).

BENITO Y DE LA LLAVE, ENRIQUE DE, "Las orientaciones modernas del Derecho Penal", Anales de la Universidad de Valencia, I-5 (1922): 380-418.

BENITO Y DE LA LLAVE, ENRIQUE DE, El problema de la responsabilidad penal de los ministros. Conferencia pronunciada en el Ateneo de Madrid en la noche del 18 de febrero de 1923, (Madrid: Gráficas reunidas, 1923).

BENITO Y DE LA LLAVE, ENRIQUE DE, "Las novedades de la legislación penal extranjera (China, Italia, Rusia, 1919-1922), Anales de la Universidad de Valencia, IV-25-32 (1923), 161-178.

Benito y DE LA LlaVe, ENRIQUe DE, "El derecho Penal Internacional y la Sociedad de Naciones", Anales de la Universidad de Valencia, VI-41-48 (1925): 96-110.

Blasco GiL, Yolanda, La Facultad de Derecho de Valencia durante la Restauración (1875-1900), (Valencia: Universitat de València, 2000).

Calonge Velázquez, Antonio, "Eduardo Callejo de la Cuesta un ministro primorriverista", Revista Aequitas, 10 (2017): 137-173.

CASSÁ BERNALDO DE Quirós, Constancio, Una pluma en el exilio. Los artículos publicados por Constancio Bernaldo de Quirós en República Dominicana, (Santo Domingo: Archivo General de la Nación, 2009).

Сово Del Rosal, Gabriela, "El proceso de elaboración del Código Penal de 1928", Anuario de Historia del Derecho, 82, (2012): 562-602.

Correa Ballester, Jorge; Francisco Javier Palao Gil, "La Facultad de Derecho de Valencia en el primer tercio del siglo XX (1900-1938)", en La enseñanza del Derecho en el siglo XX. Homenaje a Mariano Peset, ed. Adela Mora, (Madrid: Instituto Antonio de Nebrija de Estudios sobre la Universidad-Ed. Dykinson, 2004), 129-180.

FERNÁNDEZ SoRIA, JosÉ MARÍA, "Influencias nacionales europeas en la política educativa española del siglo XX", Historia de la Educación, 24 (2005): 39-95.

GARgallo VAAmonde, Luis, Desarrollo y destrucción del sistema liberal de prisiones en España. De la Restauración a la guerra civil, Tesis doctoral, (Universidad de Castilla-La Mancha, 2014). 
GutiÉRREz Gassis, Jesús, "El método de la pedagogía jurídica”, Revista de ciencias jurídicas y sociales, 15 (1921): 402-423.

HERnÁNDEZ DíAz, JosÉ MARÍA, "Nuevo derecho penal versus pedagogía correccional en Dorado Montero", en El largo camino hacia una educación inclusiva: la educación especial y social del siglo XIX a nuestros días: XV Coloquio de Historia de la Educación, Pamplona-Iruñea, 29, 30 de junio y 1 de julio de 2009, coords. María Reyes Berruezo Albéniz y Susana Conejero López, 2 vols., (Pamplona: Universidad Pública de Navarra, 2009), 125-132.

Lissorgues, Yvan, "Pensar la psicología en español en la segunda mitad del siglo XIX. (Luis Simarro Lacabra, Urbano González Serrano, Leopoldo Alas, Clarín)", en La filosofía y las lenguas de la península ibérica: Actas de las VIII y IX Jornadas Internacionales de Hispanismo filosófico. Barcelona, 2007, Santander, 2009, eds. José Luis Mora García, Ramón Emilio Mandado Gutiérrez, Gemma Gordo Piñar, Marta Nogueroles Jové, (Madrid: Fundación Ignacio Larramendi, 2010), 395-416.

LóPez Piñero, JosÉ Mạ , "La facultad de Medicina de Valencia”, en Historia de la Universidad de Valencia. Volumen III: La Universidad liberal (Siglos XIX y XX), coord. Mariano Peset, (Valencia: Universitat de València, 2000), 179-208.

Mancebo, María Fernanda, La Universidad de Valencia de Valencia. De la Monarquía a la República (1919-1939), (Valencia: Universitat de València, Instituto de Cultura Juan Gil-Albert, 1994).

MARTín, SeBASTIÁN, "Penalística y penalistas españoles a la luz del principio de la legalidad (1874-1944)", Quaderni Fiorentini per la storia del pensiero giuridico moderno, 36 (2007): 503-609.

NiÑo RodríGUEZ, ANTONIO, "El protagonismo de los intelectuales en los proyectos de reforma educativa y modernización cultural", en Modernizar España. Proyectos de reforma y apertura internacional (1898-1914), eds. Guadalupe Gómez-Ferrer y Raquel Sánchez, (Madrid: Biblioteca Nueva, 2007), 199-229.

NúÑEZ, JoRge Alberto, Fernando Cadalso y la reforma penitenciaria, (Madrid: Universidad Carlos III de Madrid, 2014).

Peset, Mariano; Jorge Correa, La Facultad de Derecho de Valencia 14991975, coords. Mariano Peset y Jorge Correa, (Valencia: Universitat de València, 2018).

Peset, Mariano; José Luis Peset, La Universidad española (siglos XVIII y XIX): Despotismo ilustrado y revolución liberal, (Madrid: Taurus, 1974). 
Ramos Vázquez, Isabel, "La Administración Civil Penitenciaria: militarismo y administrativismo en los orígenes del estado de Derecho", Anuario de Historia del Derecho Español, 82 (2012): 471-520.

RAMOS VÁzQUEZ, ISABEL La reforma penitenciaria en la historia contemporánea española, (Madrid: Dykinson, 2013).

Revista de Estudios Penitenciarios, 159 (1962).

RODRÍGUEZ FERRER, VICENTE, Manual de identificación judicial (dactiloscopia, filiación descriptiva, fotografía): reglas prácticas, (Madrid, 1914).

Ruiz-MANjón, Octavio, "Los intelectuales en la política española del primer tercio del siglo XX", en Modernizar España. Proyectos de reforma y apertura internacional (1898-1914), eds. Guadalupe Gómez-Ferrer y Raquel Sánchez, (Madrid: Biblioteca Nueva, 2007), 61-77.

Serrano Gómez, Alfonso, Historia de la Criminología en España, (Madrid: Dykinson, 2000).

Serrano Maíllo, Alfonso, Un estudio sobre la formación de la Criminología española (1903-1978). Desarrollo, hostigamiento y dimensión simbólica, (Navarra: Aranzadi, 2018).

Testor Pascual, Pascual, Discurso leído en la solemne inauguración de curso de la Universidad Literaria de Valencia por el Dr. D. Pascual Testor y Pascual, catedrático de la facultad de Derecho (1911-1912), (Valencia: Tip. Moderna, 1911).

VV.AA., Diccionario de Catedráticos Españoles de Derecho (1847-1943) (online) VV. AA., Proceso a Juan Peset Aleixandre, (Valencia: Universitat de València, 2001). 